\title{
Paramètres démographiques des cheptels de bovins Borgou et N'Dama à la Ferme d'Elevage de l'Okpara au nord-est du Bénin
}

\author{
Ibrahim Traore ALKOIRET ${ }^{1 *}$, Doha Yétongnon Gnimassou AWOHOUEDJI ${ }^{1}$ et \\ Amine Mohamed YACOUBOU ${ }^{1}$ \\ ${ }^{I}$ Département de Production Animale, Faculté d'Agronomie, Université de Parakou. \\ B.P. 123 Parakou, Bénin. \\ *Auteur correspondant ; E-mail : alkoiretib@yahoo.fr; alkouarit@gmail.com \\ Tel : 229231111 79; GSM : 22996180170 ; 229958473 84; FAX : 22923610712
}

\section{RESUME}

Les paramètres démographiques des cheptels de bovins de race Borgou et N'Dama de la Ferme d'Elevage de l'Okpara ont été étudiés de 1994 à 2008. Le cheptel Borgou a comporté une proportion élevée $(\mathrm{p}<0,05)$ de mâles $(35,3$ vs. $26,1 \%)$ et une faible $(\mathrm{p}<0,05)$ proportion de femelles $(64,7$ vs. $73,9 \%)$ par rapport au cheptel N'dama. Le taux d'avortement était plus élevé $(\mathrm{p}<0,05)$ au niveau du cheptel N'dama $(9,3$ vs. 3,9\%), alors que le taux de fécondité était au contraire plus bas $(\mathrm{p}<0,05)$ que celui du cheptel Borgou $(56,8$ vs. $69,3 \%)$. Les taux de mortalité périnatale et de mortalité globale avant sevrage du cheptel N'dama étaient plus élevés $(\mathrm{p}<0,05)$ que ceux du cheptel Borgou $(14,1$ vs. $2,2 \%$ et 5,6 vs. 2,7\% respectivement). Le cheptel Borgou avait un taux d'exploitation global significativement plus élevé (24,0 vs. 13,5\%), alors que son croît numérique net était négatif et inférieur $(\mathrm{p}<0,05)$ à celui du cheptel N'dama $(-8,8$ vs. 1,5\%). Le cheptel Borgou de cette ferme était surexploité alors que le cheptel N'dama a présenté les signes caractéristiques d'une dégénérescence consanguine.

(C) 2010 International Formulae Group. All rights reserved.

Mots clés : Pyramide des âges, fécondité, avortement, mortalité, taux d'exploitation, Bénin.

\section{INTRODUCTION}

Le cheptel bovin de la République du Bénin est estimé à 1.905.000 têtes (FAO, 2008) et la race Borgou (plus les métis Borgou-zébu) représente $88 \%$ de cet effectif (Senou et al., 2008). Cette race proviendrait d'un croisement stabilisé entre le taurin à courtes cornes d'Afrique Occidentale (Somba ou lagunaire) et le Zébu White Fulani (Domingo, 1976). Rustique, elle est dotée d'une assez bonne résistance à la trypanosomose sévissant à l'état endémique au Bénin (Doko, 1991). Son aire de répartition s'étend du Bénin au Togo, Burkina-Faso et au Nigeria où elle est connue sous le nom de Keteku (Domingo, 1976). La race N'Dama quant à elle possède une trypanotolérance au même titre que celle de la race Borgou (Doko, 1991 ; Dwinger et al., 1992 ; Knopf et al., 2002). Elle a été introduite au Bénin vers 1952 à la Ferme d'Elevage de l'Okpara dans le but d'améliorer la race bovine Borgou, mais cet essai d'amélioration s'est soldé par un échec (Youssao et al., 2000b). En 1993, le noyau de bovin N'Dama de la Ferme d'Elevage de l'Okpara est reconstitué avec l'introduction de 
22 nouvelles têtes dans le cheptel initial en dégénérescence (Youssao et al., 2000b). A ce jour, les connaissances acquises sur les races Borgou et N'Dama de la Ferme d'Elevage de l'Okpara demeurent très peu disponibles (Youssao et al., 2007; 2009). La présente étude fait partie d'une série des travaux relatifs à la caractérisation du potentiel zootechnique des bovins trypanotolérant élevés à la Ferme d'Elevage de l'Okpara. Elle a pour but de comparer les paramètres démographiques de reproduction, de viabilitémortalité et d'exploitation ainsi que la composition et la structure des cheptels bovins de races Borgou et N'Dama, élevés à la Ferme d'Elevage de l'Okpara au nord-est du Bénin.

\section{MATERIEL ET METHODES Milieu d'étude}

La Ferme d'Elevage de l'Okpara est située au nord-est du Bénin, dans le Département du Borgou et plus précisément dans la Commune de Tchaourou ( $2^{\circ} 39$ - $2^{\circ} 53$ Longitude Est et $9^{\circ} 6-9^{\circ} 21$ Latitude Nord). Elle couvre une superficie de 33.000 ha dont à peine 5.000 ha sont exploités. Le climat est de type soudanien avec l'alternance d'une saison pluvieuse (mai à octobre) et d'une saison sèche (novembre à avril). La pluviométrie annuelle est comprise entre 858 et $1.400 \mathrm{~mm}$, avec une moyenne de $1.125 \mathrm{~mm}$ enregistrée de 1994 à 2008 et la température annuelle moyenne varie entre 26 et $27^{\circ} \mathrm{C}$. Le relief est constitué d'une pénéplaine cristalline comportant des collines à roches dures. Le sol de texture sableuse, sablo-argileuse ou limoneuse par endroit supporte une végétation de savane à dominance d'Andropogon gayanus.

\section{Conduite des animaux}

Au 31 décembre 2008, le cheptel bovin (1.157 têtes) de la ferme est constitué de la race Borgou (1.015 têtes), des croisés divers (68 têtes) et de la race N'Dama (74 têtes). Les bovins N'Dama sont regroupés en un seul troupeau, alors que les Borgou sont répartis en
6 catégories de troupeaux (naisseur, génisse 1, génisse 2 , taurillon 1 , taurillon 2 et taureau reproducteur). Ces troupeaux sont logés dans des parcs de nuit disséminés sur toute la Ferme d'Elevage de l'Okpara et équipés d'abreuvoirs et de mangeoires. Le jour, de $9 \mathrm{~h}$ à $17 \mathrm{~h}$, les animaux sont conduits sur des parcours naturels et prairies artificielles constituées de Brachiaria ruziziensis, Stylosanthes sp. et Andropogon gayanus. La complémentation en ensilage, foin et tourteaux de coton a lieu pendant la période de soudure allant de janvier à avril. Les résidus de récoltes constitués de maïs, sorgho, mil et cotonnier et des ligneux fourragers sont exploités pour l'alimentation des animaux. L'eau de boisson provenant des retenus d'eau et des affluents du fleuve Okpara traversant la Ferme d'Elevage de l'Okpara, la pierre à lécher et le sel de cuisine sont servis ad libitum aux bovins. Les animaux sont soumis régulièrement à des soins préventifs de déparasitage externe et interne, de vitaminothérapie et de traitements trypanocides. Ils sont vaccinés contre la pasteurellose et la péripneumonie contagieuse bovine et sont soumis au dépistage de la brucellose et de la tuberculose. Deux montes naturelles sont organisées par an dans les troupeaux naisseurs Borgou, alors que dans le troupeau N'dama, les taureaux reproducteurs y sont laissés en permanence.

\section{Méthodologie}

Les paramètres démographiques des cheptels de bovins Borgou et N'dama de la Ferme d'Elevage de l'Okpara sont étudiés sur la base des tableaux récapitulatifs des effectifs contenus dans les rapports annuels d'activités d'une période de 15 ans allant de 1994 à 2008. Ces tableaux ont fourni des informations sur les mouvements des effectifs au cours de l'année (effectif initial, naissance, achat, mortalité, vente, don, échange, abattage et effectif final), la composition des cheptels (velle, génisse 1, génisse 2, vache, veau, bouvillon, taurillon 1 , taurillon 2 et taureau 
reproducteur), les paramètres de reproduction (gestation avancée, avortement et mise bas) et les paramètres de viabilité-mortalité (mortnés, mortalité par tranche d'âge et produits vivant au sevrage). Les Pyramides des âges des cheptels Borgou et N'Dama sont réalisées en fonction de l'âge et du sexe des animaux à la date du 30 septembre 2008. Les classes d'âge sont exprimées en pourcentage par rapport à l'effectif du cheptel de chaque race bovine.

Les paramètres démographiques annuels des cheptels de bovins Borgou et N'Dama sont calculés à l'aide des formules proposées par Lhoste et al. (1993) :

\section{Paramètres de reproduction}

Taux de fertilité apparente $=$ Nombre de gestation avancées*100 / Femelles reproductrices ;

Taux d'avortement = Nombre d'avortement*100/ Femelles reproductrices ; Taux de mise bas $=$ Nombre de mise bas*100/Femelles reproductrices ;

Taux de fécondité $=$ Produits nés vivants*100/Femelles reproductrices ;

Productivité numérique au sevrage $=$ Produits sevrés vivants*100/Femelles reproductrices ;

Productivité pondérale au sevrage $=$ Produits sevrés*Poids moyen/Femelles reproductrices, où : Poids moyen $=$ moyenne des poids au sevrage des produits sevrés au cours de l'année.

\section{Paramètres de viabilité- mortalité}

Viabilité des vaches $=100-$ (Vaches mortes/Nombre de vaches) ${ }^{*} 100$;

Taux de mortinatalité $=$ Nombre de mortnés*100/Produits nés ;

Taux de mortalité infantile endogène = Produits morts entre 0 et 15 jours*100/Produits nés ;

Taux de mortalité périnatale $=$ (mort-nés + morts entre 0 et 15 jours)*100/Produits nés ;

Taux de mortalité avant sevrage $=$ Produits morts avant sevrage*100/Produits nés vivants ;

Taux de mortalité globale $=$ Nombre total de morts*100/Effectif moyen, où :
Effectif moyen $=($ Effectif initial + Effectif final) $/ 2$.

\section{Paramètres d'exploitation numérique}

Taux d'exploitation numérique (TEN) = Animaux exploités*100/Effectif moyen ;

Croît numérique brut $(\mathrm{CNB})=($ Effectif final Effectif initial)*100/Effectif moyen ;

Croît numérique net $(\mathrm{CNN})=\mathrm{CNB}-$ (Immigration*100/Effectif moyen) ;

Rendement Numérique $(\mathrm{RN})=\mathrm{TEN}+\mathrm{CNN}$.

\section{Analyses statistiques}

Les proportions des différentes catégories d'âge et de sexe et les paramètres démographiques de reproduction, de viabilitémortalité et d'exploitation numérique des cheptels de bovins Borgou et N'dama à la Ferme d'Elevage de l'Okpara ont été calculés pour chaque année pendant la période allant de 1994 à 2008 (15 ans). Ces données ont été soumises à ANOVA (SAS, 2003) à un critère de classification (race bovine). La structuration des moyennes a été réalisée avec le test LSD (Least Significant Difference) au seuil de $5 \%$. Les résultats sont présentés sous la forme de moyennes \pm écart-types $(\mathrm{m} \pm$ ET).

\section{RESULTATS}

\section{Composition des cheptels de bovins Borgou} et N'Dama

Le cheptel Borgou a comporté plus de mâles et moins de femelles que le cheptel N'dama (Tableau 1). Le sexe ratio (mâle/femelle) a été d'un mâle pour deux femelles dans le cheptel Borgou contre un mâle pour trois femelles dans le cheptel N'Dama. Aucune différence significative ( $p>0,05)$ n'a été notée entre les deux races au niveau des proportions de velles, de génisses 1 et de vaches dans la composition des cheptels. Par contre, le cheptel N'dama a compté plus $(p<0,05)$ de génisses 2 et de femelles que le cheptel Borgou. Le cheptel Borgou a plus $(\mathrm{p}<0,05)$ de veaux, de taurillons 1 et 2 que le cheptel N'dama. Par contre, le cheptel N'dama a compté plus $(\mathrm{p}<0,05)$ de bouvillons 
et une proportion identique $(p>0,05)$ de taureaux reproducteurs. Dans la même catégorie d'âge, le cheptel N'dama a plus de velles que de veaux et plus de génisses 1 que de taurillons 1. Par contre, dans le cheptel Borgou, les proportions de velles et de veaux et celles de génisses 1 et de taurillons 1 sont presqu'identiques.

\section{Structure des cheptels de bovins Borgou et N'Dama}

Les pyramides des âges des cheptels Borgou et N'Dama en 2008 ont eu une pente régulière et ont été élargies vers la base et rétrécies vers le sommet (Figures 1 et 2 ). La pyramide des âges du cheptel Borgou a présenté une cassure au niveau de la tranche d'âge de 2 à 3 ans (Figure 1). Au niveau des deux cheptels, la tranche d'âge de 0 à 1 ans est plus petite que la tranche d'âge suivante de 1 à 2 ans.

L'âge des femelles Borgou a varié de 0 à 13 ans tandis que celui des femelles N'Dama est compris entre 0 et 11 ans (Figure 2). Moins du dixième des femelles Borgou ont plus de 6 ans, contre près du sixième chez les femelles N'Dama. Les vaches et les femelles Borgou en âge de reproduction ( 3 ans et plus) ont représenté $36 \%$ des femelles (Figure 1), contre près de la moitié pour la race N'Dama (Figure 2). La proportion de vaches Borgou âgées de 3 à 4 ans a été la plus importante parmi les femelles en âge de se reproduire, alors que les vaches N'Dama âgées de 4 à 5 ans ont été les plus nombreuses (Figure 2). Les proportions de femelles âgées de moins de trois ans ont été presqu'identiques pour les deux cheptels.

L'âge des mâles Borgou a varié de 0 à 6 ans (Figure 1), tandis que celui des mâles N'dama est compris entre 0 et 4 ans (Figure 2). Moins du dixième des mâles Borgou avaient plus de 3 ans (Figure 1) contre 1,5\% chez les mâles N'Dama (Figure 2). La proportion des mâles N'dama ayant moins d'un an a été inférieure à celle des mâles Borgou de la même tranche d'âge. De plus, l'écart entre les proportions des mâles N'dama des tranches d'âge $0-1$ et 1 - 2 ans a été le double de l'écart existant entre les mêmes tranches d'âge chez les mâles Borgou.

\section{Paramètres démographiques de reproduction des cheptels de bovins Borgou et N'Dama}

Les taux de fertilité apparente et de mise bas n'étaient pas différents $(p>0,05)$ au niveau des deux races, tandis que le taux d'avortement était plus élevé $(\mathrm{p}<0,05)$ dans le cheptel N'dama (Tableau 2). Ceci a eu une répercussion sur le taux de fécondité et la productivité numérique au sevrage de ce cheptel. Ces deux paramètres étaient en effet plus élevés $(p<0,05)$ au niveau de la race Borgou. La productivité pondérale au sevrage du cheptel Borgou a été aussi meilleure $(p<0,05)$ que celle du cheptel N'dama.

\section{Paramètres de viabilité-mortalité des cheptels de bovins Borgou et N'dama}

La viabilité des vaches était identique ( $p>0,05)$ et élevée au niveau des deux races. Le taux de mortalité des jeunes était aussi identique $(p>0,05)$ et relativement faible dans les deux cheptels (Tableau 3). Par contre, le nombre de veaux mort-nés et morts entre la naissance et l'âge de 15 jours était plus élevé $(\mathrm{p}<0,05)$ dans le cheptel N'dama. Ainsi, le taux de mortalité périnatale était plus élevé $(\mathrm{p}<0,05)$ dans ce cheptel.

La mortalité des velles était identique $(\mathrm{p}>0,05)$ dans les deux races, alors que la mortalité des veaux de race N'dama était plus élevée $(\mathrm{p}<0,05)$ que celle des veaux Borgou. Ceci a induit un taux de mortalité globale avant sevrage plus élevé $(\mathrm{p}<0,05)$ au niveau du cheptel N'dama. Le taux de mortalité global était plus élevé $(\mathrm{p}<0,05)$ au niveau du cheptel N'dama par rapport au cheptel Borgou.

\section{Paramètres d'exploitation numérique des cheptels de bovins Borgou et N'Dama}

Les taux d'exploitation des femelles et 
mâles de race Borgou sont supérieurs $(\mathrm{p}<0,05)$ à ceux de la race N'dama (Tableau 4). Il en a résulté un taux d'exploitation global plus élevé $(\mathrm{p}<0,05)$ du cheptel Borgou. Dans l'ensemble et au niveau des cheptels Borgou et N'dama, l'exploitation des femelles était plus faible que celle des mâles. Le rapport de l'exploitation des mâles sur celle des femelles était de 1,8 pour la race Borgou et de 2,4 pour la race N'dama. Ainsi, les mâles du cheptel N'dama étaient plus exploités que ceux du cheptel Borgou. Les croîts numériques brut et net $\mathrm{du}$ cheptel Borgou étaient négatifs et inférieurs $(\mathrm{p}<0,05)$ à ceux du cheptel $\mathrm{N}$ 'dama qui étaient identiques. Les rendements numériques des cheptels Borgou et N'dama étaient identiques $(p>0,05)$ pendant toute la période d'observation. Le rendement numérique du cheptel Borgou était inférieur à son taux d'exploitation numérique, alors que l'effet contraire était observé au niveau du cheptel N'dama.

Tableau 1 : Composition $(\mathrm{m} \pm \mathrm{ET})$ des cheptels de bovins Borgou et N'Dama à la Ferme d'Elevage de l'Okpara.

\begin{tabular}{lcc}
\hline Composition & Bovin Borgou (\%) & Bovin N'dama (\%) \\
\hline Femelles & $12,07 \pm 4,64^{\mathrm{a}}$ & \\
Velles & $16,17 \pm 2,74^{\mathrm{a}}$ & $11,45 \pm 3,44^{\mathrm{a}}$ \\
Génisses 1 (âgées de 1 à 2 ans) & $7,16 \pm 2,76^{\mathrm{a}}$ & $15,85 \pm 4,61^{\mathrm{a}}$ \\
Génisses 2 (âgées de 2 à 3 ans) & $29,31 \pm 6,39^{\mathrm{a}}$ & $14,31 \pm 6,81^{\mathrm{b}}$ \\
Vaches & $64,71 \pm 5,43^{\mathrm{a}}$ & $32,34 \pm 4,72^{\mathrm{a}}$ \\
Total des femelles & & $73,94 \pm 6,85^{\mathrm{b}}$ \\
Mâles & $11,85 \pm 4,15^{\mathrm{b}}$ & \\
Veaux & $1,32 \pm 0,99^{\mathrm{a}}$ & $7,43 \pm 2,86^{\mathrm{a}}$ \\
Bouvillons & $14,28 \pm 4,72^{\mathrm{b}}$ & $4,03 \pm 2,75^{\mathrm{b}}$ \\
Taurillons 1 (âgés de 1 à 2 ans) & $6,51 \pm 4,68^{\mathrm{b}}$ & $9,8 \pm 4,63^{\mathrm{a}}$ \\
Taurillons 2 (âgés de 2 à 3 ans) & $1,33 \pm 0,62^{\mathrm{a}}$ & $3,12 \pm 1,78^{\mathrm{a}}$ \\
Taureaux & $35,29 \pm 5,43^{\mathrm{b}}$ & $1,65 \pm 0,57^{\mathrm{a}}$ \\
Total des mâles & 100 & $26,06 \pm 6,85^{\mathrm{a}}$ \\
Total & & 100 \\
\hline
\end{tabular}

Les valeurs moyennes sur une même ligne surmontées des lettres différentes a, b et c sont significativement différentes $(\mathrm{p}<0,05)$.

Tableau 2 : Paramètres démographiques de reproduction $(\mathrm{m} \pm \mathrm{ET})$ des cheptels de bovins Borgou et N'Dama à la Ferme d'Elevage de l'Okpara.

\begin{tabular}{lcc}
\hline Paramètres & Bovins Borgou (\%) & Bovins N'dama (\%) \\
\hline Taux de fertilité apparente & $74,80 \pm 13,63^{\mathrm{a}}$ & $70,44 \pm 16,42^{\mathrm{a}}$ \\
Taux d'avortement & $3,93 \pm 1,28^{\mathrm{a}}$ & $9,31 \pm 3,49^{\mathrm{b}}$ \\
Taux de mise bas & $70,27 \pm 15,23^{\mathrm{a}}$ & $61,13 \pm 19,08^{\mathrm{a}}$ \\
Taux de fécondité & $69,28 \pm 13,06^{\mathrm{b}}$ & $56,83 \pm 15,35^{\mathrm{a}}$ \\
Productivité numérique au sevrage & $67,33 \pm 12,76^{\mathrm{b}}$ & $53,73 \pm 13,74^{\mathrm{a}}$ \\
Productivité pondérale au sevrage $(\mathrm{kg})$ & $68,41 \pm 11,83^{\mathrm{b}}$ & $52,23 \pm 13,14^{\mathrm{a}}$
\end{tabular}

Les valeurs moyennes sur une même ligne surmontées des lettres différentes a, b et c sont significativement différentes $(\mathrm{p}<0,05)$. 
Tableau 3 : Paramètres démographiques de viabilité-mortalité $(\mathrm{m} \pm \mathrm{ET})$ des cheptels de bovins Borgou et N'Dama à la Ferme d'Elevage de l'Okpara.

\begin{tabular}{lcc}
\hline Paramètres & Bovins Borgou (\%) & Bovins N'dama (\%) \\
\hline Viabilité des vaches & $99,79 \pm 0,20^{\mathrm{a}}$ & $99,74 \pm 0,91^{\mathrm{a}}$ \\
Taux de mortinatalité & $1,52 \pm 0,70^{\mathrm{a}}$ & $9,91 \pm 4,32^{\mathrm{b}}$ \\
Taux de mortalité infantile endogène & $0,70 \pm 0,49^{\mathrm{a}}$ & $4,17 \pm 2,57^{\mathrm{b}}$ \\
Taux de mortalité périnatale & $2,22 \pm 1,20^{\mathrm{a}}$ & $14,08 \pm 6,68^{\mathrm{b}}$ \\
Taux de mortalité des velles & $2,73 \pm 1,43^{\mathrm{a}}$ & $2,40 \pm 1,88^{\mathrm{a}}$ \\
Taux de mortalité des veaux & $2,57 \pm 1,38^{\mathrm{a}}$ & $9,95 \pm 3,87^{\mathrm{b}}$ \\
Taux de mortalité globale avant sevrage & $2,67 \pm 1,42^{\mathrm{a}}$ & $5,58 \pm 2,28^{\mathrm{b}}$ \\
Taux de mortalité des jeunes & $0,98 \pm 0,43^{\mathrm{a}}$ & $0,99 \pm 0,52^{\mathrm{a}}$ \\
Taux de mortalité des adultes & $0,82 \pm 0,68^{\mathrm{b}}$ & $0,33 \pm 0,19^{\mathrm{a}}$ \\
Taux de mortalité globale & $1,86 \pm 0,76^{\mathrm{a}}$ & $2,64 \pm 1,08^{\mathrm{b}}$ \\
\hline
\end{tabular}

Les valeurs moyennes sur une même ligne surmontées des lettres différentes $a, b$ et $c$ sont significativement différentes $(p<0,05)$

Tableau 4 : Paramètres d'exploitation numérique $(\mathrm{m} \pm \mathrm{ET})$ des cheptels de bovins Borgou et N'Dama à la Ferme d'Elevage de l'Okpara.

\begin{tabular}{lcc}
\hline Paramètres & Bovins Borgou (\%) & Bovins N'dama (\%) \\
\hline Taux d'exploitation femelles & $19,05 \pm 6,88^{\mathrm{b}}$ & $9,80 \pm 5,25^{\mathrm{a}}$ \\
Taux d'exploitation mâles & $35,32 \pm 16,02^{\mathrm{b}}$ & $23,57 \pm 11,54^{\mathrm{a}}$ \\
Taux d'exploitation global & $23,95 \pm 6,97^{\mathrm{b}}$ & $13,46 \pm 7,22^{\mathrm{a}}$ \\
Croît numérique brut & $-7,38 \pm 4,17^{\mathrm{a}}$ & $1,47 \pm 8,11^{\mathrm{b}}$ \\
Croît numérique net & $-8,75 \pm 4,28^{\mathrm{a}}$ & $1,47 \pm 8,11^{\mathrm{b}}$ \\
Rendement numérique & $15,20 \pm 6,64^{\mathrm{a}}$ & $14,85 \pm 6,24^{\mathrm{a}}$ \\
\hline
\end{tabular}

Les valeurs moyennes sur une même ligne surmontées des lettres différentes $a, b$ et $c$ sont significativement différentes $(p<0,05)$.
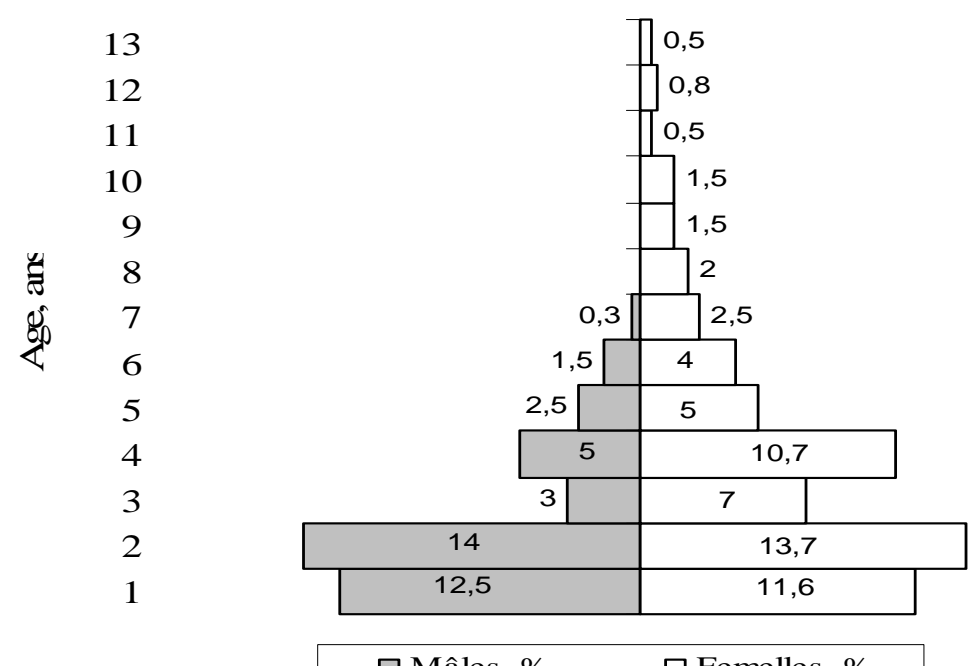

Figure 1 : Pyramide des âges du cheptel Borgou à la Ferme d'Elevage de l'Okpara en 2008. 


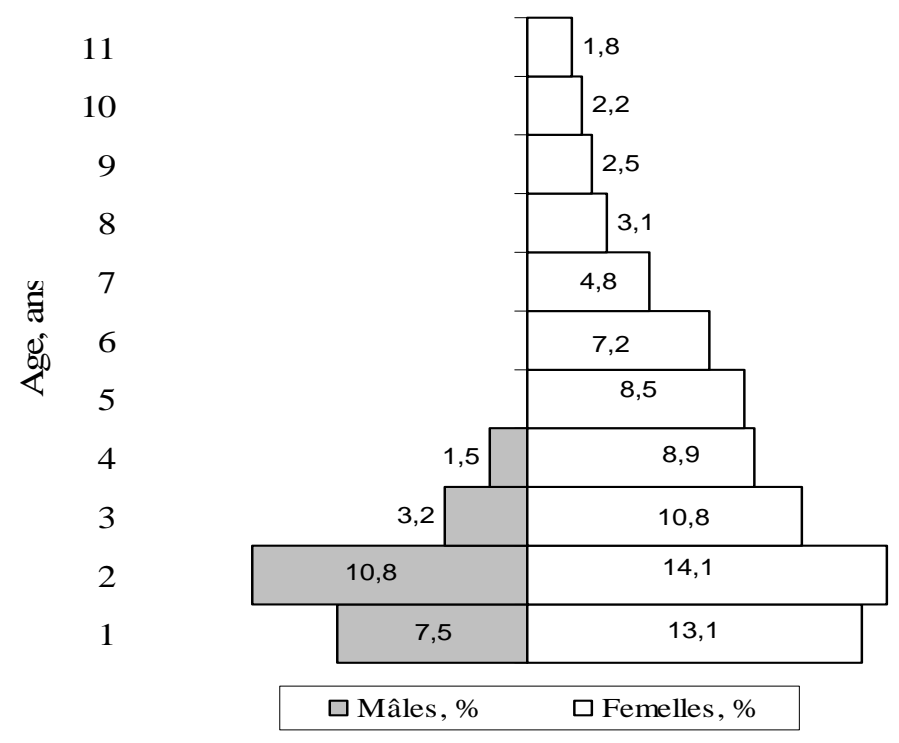

Figure 2 : Pyramide des âges du cheptel N'Dama à la Ferme d'Elevage de l'Okpara en 2008.

\section{DISCUSSION}

La composition et la structure du cheptel Borgou de la Ferme d'Elevage de l'Okpara sont différentes de celles du cheptel N'dama et ne correspondent pas au constat fait par Dehoux et Hounsou-Ve (1993) dans les troupeaux bovins traditionnels installés dans le département du Borgou au nord-est du Bénin. En effet, les troupeaux bovins traditionnels comptent un mâle pour trois femelles car ce type d'élevage traditionnel conserve de vieilles vaches productives ou non et toutes les génisses de réforme (Dehoux et Hounsou-Ve, 1993). Ainsi, dans les élevages transhumants, $18,2 \%$ des vaches ont plus de 9 ans contre $12,7 \%$ dans les élevages sédentaires (Dehoux et Hounsou-Ve, 1993). L'éleveur traditionnel est intéressé par l'effectif de son cheptel qui représente sa richesse et suscite son admiration et sa fierté (Youssao et al., 2000a ; Ezanno et al., 2002). Une telle pratique explique la proportion élevée de vaches de plus de 6 ans et de génisses 2 dans le cheptel N'dama et la concordance de la composition de ce troupeau avec celle des troupeaux traditionnels. La proportion de vaches dans les cheptels Borgou et N'dama de la Ferme d'Elevage de l'Okpara est inférieure à celle des troupeaux traditionnels du département du Borgou (Dehoux et Hounsou-Ve, 1993) et des troupeaux de bovins Horro en Ethiopie (Lesnoff et al., 2002), comportant $40 \%$ de femelles reproductrices. A la Ferme d'Elevage de l'Okpara, les génisses et taurillons Borgou de $1^{\text {er }}$ âge ne sont pas tous mutés dans les troupeaux de $2^{\text {ème }}$ âge. Avant la mutation en $2^{\text {ème }}$ âge, près du tiers des animaux de $1^{\text {er }}$ âge est éliminé pour insuffisance de poids et défauts des aplombs, ce qui explique la proportion réduite des animaux de la tranche d'âge de 2 à 3 ans dans la structure de ce cheptel. Dans les élevages bovins sédentaires ou transhumants, la proportion de veaux est de $16,7 \%$ et celle de velles de 16,4\% (Dehoux et Hounsou-Ve, 1993) et ces proportions sont supérieures à celles enregistrées dans les cheptels bovins de race Borgou et N'dama de la Ferme d'Elevage de l'Okpara. La proportion élevée des veaux de race Borgou par rapport à celle des veaux de race N'dama est liée à la forte mortalité des veaux N'dama.

Les paramètres de reproduction du cheptel Borgou sont supérieurs à ceux du cheptel N'dama de la Ferme d'Elevage de l'Okpara et s'expliquent par la détérioration 
des performances de reproduction des animaux de race N'dama. En effet, huit ans plus tôt, le troupeau N'dama de la Ferme d'Elevage de l'Okpara a eu un taux de fécondité supérieur de 6,8\% par rapport au taux de fécondité du cheptel Borgou (Youssao et al., 2000a, 2000b). Par conséquent, dans notre étude, la baisse du taux de fécondité du troupeau N'dama est liée à la hausse du taux d'avortement. Pourtant, les bovins N'dama sont réputés pour une bonne fécondité avec des taux supérieurs à 85\% (Colomb, 1976 ; Khang'mate et al., 2000 ; Ezanno et al., 2002). Le taux moyen de fécondité du cheptel Borgou de la Ferme d'Elevage de l'Okpara est plus élevé que les taux de fécondité de 64,4 et $66,9 \%$ enregistrés dans les élevages bovins traditionnels sédentaires et transhumants (Dehoux et Hounsou-Ve, 1993). Par contre ce taux est inférieur au taux de fécondité des zébus Azaouak de la station de Toukounous qui s'élève à 78\% (Achard et Chanono, 1997).

Les taux de mortalité global des cheptels Borgou et N'dama à la Ferme d'Elevage de l'Okpara sont inférieurs à celui de 4,9\% établi à la station de Toukounous au Niger (Achard et Chanono, 1997). Les taux de mortalité des jeunes et des adultes à 1'Okpara sont inférieurs à ceux de 9 et $3 \%$ trouvés dans les troupeaux bovins de race Horro en Ethiopie (Lesnoff et al., 2002). En milieu traditionnel du Sénégal, Ezanno et al. (2002) ont établi des probabilités de mort de 10 et $2 \%$ pour les bovins N'dama jeunes (1-2 ans) et adultes. Les taux de mortalité avant sevrage des cheptels Borgou et N'dama à la Ferme d'Elevage de l'Okpara sont aussi inférieurs à ceux enregistrés par Achard et Chanono (1997) à la station de Toukounous (8,5\%), par Lesnoff et al. (2002) en milieu traditionel en Ethiopie (17\%), par Knopf et al. (2002) en milieu traditionnel en Côte d'Ivoire (19\%) et par Youssao et al. (2000a) en milieu traditionnel au Bénin (23\%). Le taux de mortalité avant sevrage du cheptel N'dama de l'Okpara est comparable à celui de $6 \%$ trouvé par Ganaba et al. (2002) au Burkina-Faso. Hormis les taux de mortalité des jeunes et des adultes, les paramètres de mortalité du cheptel
N'dama de la Ferme d'Elevage de l'Okpara sont supérieurs à ceux du cheptel Borgou. Selon Youssao et al. (2000b), le cheptel N'dama avait de faibles paramètres de mortalité au début de la période d'observation qui ont considérablement augmenté par la suite. Pourtant les animaux des deux races sont soumis au même programme de prophylaxie. La baisse des performances de production et de reproduction suivie de la hausse de la mortalité périnatale et juvénile sont les signes caractérisant la dégénérescence d'un troupeau à faible effectif soumis à une dépression consanguine (Minvielle, 1990; Verrier, 1992 ; Colleau et Moureaux, 2006). Nous supposons que le troupeau N'dama de la Ferme d'Elevage de l'Okpara n'ayant bénéficié d'aucun apport de sang nouveau depuis 1993 et où la monte est libre est en pleine dégénérescence consanguine.

Le cheptel Borgou de la Ferme d'Elevage de l'Okpara est plus exploité certainement grâce à son grand effectif par rapport à celui du troupeau N'dama et aussi par souci de conservation et de sauvegarde de ce patrimoine animale, reconnu sur le plan mondial comme une ressource génétique précieuse. La race N'dama est identifiée comme étant la plus résistante aux trypanosomes africains pathogènes (Doko, 1991 ; Dwinger et al., 1992 ; Knopf et al., 2002). Néanmoins, les mâles N'dama sont plus exploités car ils sont très tôt castrés pour éviter la consanguinité et sont utilisés pour la boucherie. Que ce soit dans le cheptel Borgou ou N'dama, le taux d'exploitation des mâles est supérieur à celui des femelles. Ce constat est en concordance avec les observations de Dehoux et Hounsou-Ve (1993) et Youssao et al. (2000a) où l'exploitation numérique varie beaucoup selon les catégories de bétail, les mâles étant vendus très jeunes, alors que les femelles sont conservées longtemps pour la reproduction. Le signe négatif des croîts numériques brut et net du cheptel Borgou signifie que l'exploitation de ce cheptel dépasse ses capacités reproductrices. Les animaux de race Borgou prélevés ne sont pas tous remplacés et l'effectif de ce cheptel a 
baissé de 1994 à 2008, alors que l'effectif du cheptel N'dama a légèrement augmenté. $\mathrm{Au}$ niveau du cheptel N'dama, les croîts numériques brut et net sont égaux, montrant que ce cheptel n'a bénéficié d'aucun apport numérique extérieur pendant cette même période.

\section{Conclusion}

Notre étude montre que la composition et la structure du cheptel Borgou de la Ferme d'Elevage de l'Okpara est différente de celles du cheptel N'dama. Le cheptel Borgou a un taux de fécondité et une productivité numérique et pondérale au sevrage plus élevé, ainsi que des taux d'avortement et de mortalité globale avant sevrage plus faible que ceux de la race N'dama.

Le cheptel Borgou est soumis à une forte exploitation numérique qui inhibe son croît numérique, alors que le cheptel N'dama est caractérisé par une faible fécondité et une forte mortalité périnatale et juvénile révélatrice d'un taux élevé de consanguinité.

Les responsables de la Ferme d'Elevage de l'Okpara doivent prendre des mesures urgentes pour baisser l'exploitation $\mathrm{du}$ cheptel Borgou afin de le stabiliser ou d'accroître son effectif et pour introduire du sang nouveau dans le troupeau N'dama qui est en pleine dégénérescence consanguine.

\section{REMERCIEMENTS}

Les auteurs remercient le personnel de la FEO pour leur franche et précieuse collaboration, et le directeur du Projet de Développement de l'Elevage phase III pour avoir autorisé cette étude.

\section{REFERENCES}

Achard F, Chanono M. 1997. Mortalité et performances de reproduction chez le zébu Azaouak à la station de Toukounous, Niger (1986-1992). Revue Elev. Méd. Vét. Pays Trop. 50(4): 325333.

Colleau J-J, Moureaux S. 2006. Gestion optimisée de la parenté et de la consanguinité dans les programmes de sélection des bovins laitiers. INRA, Prod. Anim., 19(1): 3-14.

Coulomb J. 1976. La race N'Dama. Quelques caractéristiques zootechniques. Revue Elev. Méd. Vét. Pays Trop., 29: 367-380.

Dehoux J-P, Hounsou-Ve G. 1993. Productivité de la race bovine Borgou selon les systèmes d'élevage traditionnels au nord-est du Bénin. Revue Mondiale de Zootechnie, 74/ 75: 36-48.

Doko AS. 1991. Etude sur la trypanosomiase et la trypanotolérance bovines au Bénin. Thèse M.Sc., Institut de Médecine Tropicale, Anvers, Belgique, p. 94.

Domingo A. 1976. Contribution à l'étude de la population bovine des Etats du Golfe du Bénin. Thèse Doct. vét., Ecole interEtats des sciences et médecine vétérinaires, Dakar, Sénégal, p. 143.

Dwinger RH, Clifford DJ, Agyemang K, Gettinby G, Grieve AS, Kora S, Bojang MA. 1992. Comparative studies on N'Dama and zebu cattle following repeated infections with Trypanosoma congolense. Res. Vet. Sci., 52: 292-298.

Ezanno P, Ickowicz A, Faye B. 2002. Demographic parameters of N'dama cattle raised under extensive range management conditions in southern Senegal. Revue Elev. Méd. Vét. Pays Trop., 55(3): 211-219.

FAO. 2008. Données statistiques de la FAO (FAO-STAT). Consulté le 24 septembre 2010 à 16 h sur le site http://faostat.fao. org/DesktopDefault.aspx?

Ganaba R, Bengaly Z, Ouattara L. 2002. Calf morbidity, mortality and parasite prevalence in the cotton zone of Burkina Faso. Prev. Vet. Med., 55: 209-216.

Khang'Mate AB, Lahlou-Kassi A, Bakana BM, Kahungu M. 2000. Performances de reproduction des bovins N'dama dans le diocèse d'Idiofa au Congo. Revue Mèd. Vét., 151(6): 511-516.

Knopf L, Komoin-oka C, Betschart B, Jongejan F, Gottstein B, Zinsstag J. 2002. Seasonal epidemiology of ticks and aspects of cowdriosis in N'Dama village 
cattle in the Central Guinea savannah of Côte d'Ivoire. Prev. Vet. Med., 53: 21-30.

Lesnoff M, Diedhiou M, Laval G, Bonnet P, Workalemahu A, Kifle D. 2002. Demographic Parameters of a Domestic Cattle Herd in a Contagious-BovinePleuropneumonia Infected Area of Ethiopian Highlands. Revue Elev. Méd. Vét. Pays Trop., 55(2): 139-147.

Lhoste P, Dolle V, Rousseau J, Soltner D. 1993. Manuel de Zootechnie des Régions Chaudes : les Systèmes d'Elevage. Collection Précis d'Elevage: France ; 281.

Minvielle F. 1990. Principes d'Amélioration Génétique des Animaux Domestiques. INRA : Paris ; 211.

SAS. 2003. SAS user's Guide: Statistics, Version 9.1. Carry, NC, USA. Statistical Analysis System Inc.

Senou M, Toleba SS, Adandedjan C, Poivey J-P, Ahissou A, Toure ZF, Monsia C. 2008. Increased milk yield in Borgou cows in alternative feeding systems. Revue Elev. Méd. Vét. Pays Trop., 61(2): 109-114.

Verrier E., 1992. La gestion génétique des petites populations. In: Eléments de génétique quantitative et application aux populations animales. INRA, Prod. Anim., hors série : 265-271.
Youssao AKI, Ahissou A, Toure Z, Leroy PL. 2000a. Productivité de la race bovine Borgou à la ferme d'élevage de l'Okpara au Bénin. Revue Elev. Méd. Vét. Pays Trop., 53(1): 67-74.

Youssao AKI, Ahissou A, Toure Z. 2000b. Introduction de la race bovine N'Dama à la Ferme Elevage de l'Okpara au Bénin. Quelques performances zootechniques. Animal Genetic Resources Information, 27: 17-25.

Youssao AKI, Koutinhouin GB, Kpodekon TM, Agnandjo H, Toure Z, Ahissou A, Renand G. 2007. Variabilité génétique des performances de croissance et des mesures corporelles de jeunes bovins de race Borgou à la Ferme d'Elevage de l'Okpara. Revue Africaine de Santé et de Productions Animales, 5(3-4): 157-165.

Youssao AKI, Koutinhouin GB, Kpodekon TM, Agnandjo H, Toure Z, Ahissou A. 2009. Influence d'une sélection phénotypique sur les performances de croissance et les caractères de développements musculaire et squelettique de jeunes bovins de race Borgou à la Ferme d'Elevage de l'Okpara (Bénin). Ann. Méd. Vét., 153: 105-111. 